\title{
Beiträge der Tourismusgeographie zur Klimaänderungsfolgenforschung
}

\section{Einleitung}

Die Klimaänderung und die globale Erwärmung zählen zweifellos zu den wichtigsten Umweltproblemen. Es erstaunt deshalb nicht, daß in den letzten Jahren und Jahrzehnten die Klimaforschung und die Klimafolgenforschung einen zunehmend größeren Stellenwert erlangt haben. Auf der einen Seite geht es dabei um die Erforschung der komplexen Ursachen und Mechanismen von Klimaänderungen mit dem Ziel, Aussagen über künftige Klimaänderungen auf globaler und regionaler Ebene machen zu können. Anderseits geht es aber immer mehr auch darum, mögliche Folgen eines künftigen Klimawandels abzuschätzen und entsprechende Anpassungs- und Vermeidungsstrategien sowie Maßnahmen zu entwickeln. Die Klimafolgenforschung beschäftigt sich generell mit dem Zusammenhang zwischen dem Klima einerseits sowie den natürlichen und anthropogenen Elementen unseres Lebensraumes anderseits; als Beispiel dafür können Klimaeignungsstudien genannt werden. Heute wird der Begriff vor allem im Zusammenhang mit der Klimaänderung verwendet; korrekterweise müßte man dann allerdings von «Klimaänderungsfolgenforschung» sprechen.

$\mathrm{Zu}$ Beginn der Klimaänderungsfolgenforschung richtete sich das Augenmerk hauptsächlich auf naturräumliche Systeme, wie die Vegetation, den Wasserhaushalt oder die Kryosphäre. Die Auswirkungen auf sozioökonomische Systeme werden erst seit jüngerer Zeit näher analysiert. Im Mittelpunkt standen hier zuerst die Auswirkungen auf die Landwirtschaft und die Nahrungsmittelproduktion. Neben der Landwirtschaft zählt zweifellos der Tourismus zu denjenigen Wirtschaftszweigen, die in starkem Maße von Wetter und Klima geprägt werden. Trotz der großen Bedeutung des Klimas für den Tourismus muß jedoch, bereits an dieser Stelle, vor einem Klimadeterminismus gewarnt werden. Eine Vielzahl weiterer Einflußgrößen ist für die touristische Entwicklung mit verantwortlich, auch wenn von seiten der Touristiker oft dem Klima die Schuld für schlechte Betriebsergebnisse zugeschoben wird.

In den letzten Jahren wurden in der Schweiz mit ihrer großen volks- und regionalwirtschaftlichen Abhängigkeit vom Tourismus die Anstrengungen im Bereich der touristisch orientierten Klimafolgenforschung intensiviert. Gemessen an den Forschungsanstrengungen im Bereich der eigentlichen Klimaforschung (Klimamodellierungen usw.), sind sie allerdings immer noch sehr bescheiden. Seit rund sechs Jahren beschäftigen wir uns am Geographischen Institut der Universität Zürich (Abt. Wirtschaftsgeographie) mit Auswirkungen von Klimaänderungen auf den Tourismus im schweizerischen Berggebiet, insbesondere auf den alpinen Wintertourismus, sowie mit entsprechenden Untersuchungen in den australischen Alpen. Es handelt sich dabei um Untersuchungen, die zu einem wesentlichen Teil im Rahmen des Nationalen Forschungsprogramms «Klimaänderungen und Naturkatastrophen» (NFP 31) durchgeführt wurden und im Rahmen des Schwerpunktprogramms «Umwelt» (SPPU) weitergeführt werden.

In seinem Aufsatz «Klimawandel, Klimawirkungsforschung und die Rolle der Geographie» weist der deutsche Geograph und Klimaforscher STERR (1993) darauf hin, daß die Geographie als klassisches Brückenfach zwischen der Geo- und der Anthroposphäre wichtige Beiträge zur Klimafolgenforschung leisten kann. Aus unserer Sicht ist jedoch der Beitrag der Geographie, insbesondere der Tourismusgeographie, in diesem aktuellen und attraktiven Forschungsgebiet vorläufig noch zu gering. Das Plädoyer für ein größeres Engagement der Geographie bedeutet aber nicht, daß eine monodisziplinäre Betrachtungsweise unterstützt werden soll. Es geht vielmehr um eine verstärkte Mitwirkung der Geographie in transdisziplinären Forschungsprojekten.

\section{Zur Geschichte der Klimafolgenforschung}

Nach TIMMERMAN (1989) kann die bisherige Forschung über Wirkungen des Klimas auf sozioökonomische Systeme in drei Phasen unterteilt werden:

1. Die erste Phase bis ca. Mitte der sechziger Jahre ist geprägt durch a) klimadeterministische Ansätze und b) Untersuchungen der Auswirkungen einzelner Klimaund Wetterereignisse.

Hans Elsasser, Prof. Dr., und Rolf Bürki, dipl. geogr., Geographisches Institut der Universität Zürich

Bruno Abegg, Dr., Zürich

Urs König, Dr., Victoria University of Technology, Faculty of Business, Melbourne AUS 
a) Als Beispiele für den Klimadeterminismus können genannt werden:

E. Brückner (1910): Klimaschwankungen und Völkerwanderungen im 19. Jahrhundert

E. Huntington (1915): Civilisation and Climate

W. Hellpach (1923): Die geopsychischen Erscheinungen Wetter und Klima, Boden und Landschaft in ihrem Einfluß auf das Seelenleben

K. Sapper (1941): Der Wirtschaftsgeist und die Arbeitsleistung tropischer Kolonialvölker

G. Grundke (1955): Die Bedeutung des Klimas für den industriellen Standort

b) In dieser ersten Phase begann man sich auch vermehrt mit den Auswirkungen räumlich meist eng begrenzter Klimaereignisse (Naturkatastrophen) wie Überschwemmungen, Murgänge, Lawinenniedergänge oder Dürren auseinanderzusetzen. Aus der deutschsprachigen geographischen Tourismusforschung können hier beispielhaft folgende Arbeiten, die allerdings aus den siebziger Jahren stammen, genannt werden:

Chr. Hannss (1975): Die Lawinen- und Überschwemmungsschäden in der Wintersportstation Vald'Isère, Hochtarentaise

Th. Pippan (1977): Der Einfluß von katastrophalen Wetterereignissen auf den Saison-Fremdenverkehr und die behördlichen Kontrollmaßnahmen am Beispiel des Landes Salzburg

2. Zwischen Mitte der sechziger und Mitte der achtziger Jahre wurden diese Wirkungsstudien ausgeweitet. Einerseits wurden einzelne Klimaereignisse in den größeren Zusammenhang der Klimaänderung gestellt, d.h., die Klimafolgenforschung entwickelte sich immer mehr zu einer Klimaänderungsfolgenforschung, und anderseits wurden die Auswirkungen in einen umfassenderen sozioökonomischen Kontext gestellt. Wertvolle Beiträge wurden vermehrt auch von Historikern geliefert, z. B. über Ursachen und Auswirkungen von Hungersnöten. Da es sich beim Massentourismus um ein junges Phänomen handelt, erstaunt es nicht, daß entsprechende historische Untersuchungen fehlen. Eine Untersuchung, die den vermuteten Zusammenhang zwischen Wirtschaftsboom, schneereichen Wintern und der rasanten Entwicklung des Wintertourismus in den Alpen in den sechziger und siebziger Jahren analysiert, fehlt leider noch.

3. Die dritte Phase, die vor gut zehn Jahren begann, ist geprägt durch Untersuchungen über die Auswirkungen der globalen Erwärmung auf komplexe sozioökonomische Systeme. Einerseits werden die Klimamodelle laufend verbessert sowie räumlich und zeitlich verfeinert, anderseits werden die Rückkoppelungen innerhalb der sozioökonomischen Systeme sowie zwischen Klima, Umwelt, Wirtschaft, Gesellschaft und Politik mit berücksichtigt. In diesem Zusammenhang ist auf das deutsche Bund-Länder-Forschungsprogramm «Klimaänderung und Küste» hinzuweisen, in welchem unter dem Titel «Küstentourismus und Klimawandel» die
Entwicklung des Tourismus im deutschen Küstenbereich unter besonderer Berücksichtigung der Wahrnehmung und Bewertung von Klimafolgen durch relevante Entscheidungsträger speziell untersucht wird.

\section{Vom einfachen «impact approach» zur ganzheitlichen Klimafolgenforschung}

Der einfache «impact approach» kann an einem touristischen Beispiel folgendermaßen dargestellt werden: Höhere Durchschnittstemperaturen

$\rightarrow$ weniger Schnee (first-order impact)

$\rightarrow$ weniger Skifahrer (second-order impact).

Es wird also versucht abzuschätzen, wie groß die Zahl der Skifahrer sein wird, wenn sich die Durchschnittstemperaturen erhöhen, wobei alle anderen Faktoren, die ebenfalls die Zahl der Skifahrer beeinflussen können, konstant gehalten werden. Auch wenn die (vorläufige) Nichtberücksichtigung weiterer Bestimmungsfaktoren akzeptiert wird, sind wir bereits bei diesem Ansatz mit einer Vielzahl von Problemen konfrontiert:

Das Hauptproblem beim «impact approach» ist, daß man von Veränderungen im Klima über Veränderungen in den naturräumlichen Voraussetzungen direkt auf Veränderungen in Wirtschaft und Gesellschaft schließt. Dieser Ansatz besitzt somit eine klimadeterministische Komponente.

Klimamodelle liefern in den meisten Fällen «lediglich» Mittelwerte einzelner Klimaparameter, oft nur für Temperatur und Niederschlag. Gerade im Tourismus läßt sich die Bedeutung von Klima und Wetter nicht auf mittlere Temperaturen und Niederschlagssummen reduzieren. Mittelwerte bei Klimaänderungen, beispielsweise eine Erhöhung der Durchschnittstemperatur um 1 Grad Celsius und eine damit verbundene Verschiebung der Höhengrenze der Schneesicherheit um $150 \mathrm{~m}$ nach oben, was gemäß IPPC-Szenario A («business as usual») ungefähr im Jahre 2025 eintreten könnte, sind häufig geringer als die jährlichen Schwankungen. Die natürliche Variabilität des Klimas wird oft vergessen. Diese Vernachlässigung der Klimavariabilität betrifft aber nicht allein den «impact approach», sondern auch die beiden folgenden Ansätze. Die Schwierigkeit, Touristiker bezüglich der Probleme einer Klimaänderung zu sensibilisieren, findet bei der Klimavariabilität eine Ursache, denn es gab und gibt allein aufgrund der natürlichen Variabilität des Klimas immer wieder wärmere, schneearme und kältere, schneereiche Winter. Während in der Schweiz die Sensibilisierung der Tourismusverantwortlichen relativ hoch ist, ist für $90 \%$ der ski resort manager in den australischen Alpen die Klimänderung kein Thema, das es beim Betrieb und der Planung in den Skistationen zu berücksichtigen gilt. Sie betrachten vielmehr die entsprechenden Diskussionen in den Medien als eine Gefahr für ihr Geschäft.

Realitätsnäher ist der sog. interaktive Ansatz («interactive approach»). Er berücksichtigt - um beim obigen 
Beispiel zu bleiben -, daß die Zahl der Skifahrer in einem Skigebiet nicht allein von den Schneeverhältnissen abhängig ist, sondern daß eine Vielzahl externer Faktoren eine Rolle spielt, wie beispielsweise die Tourismuspolitik, Modeströmungen im Tourismus u. ä.m. Ein Beispiel dazu: Gemäß der MACH-Consumer-Studie der AG für Werbemedienforschung (WEMF) fuhren 1992 von den 14- bis 19jährigen Schweizerinnen und Schweizern $65 \%$ Ski und 12\% Snowboard; 1997 lauteten die entsprechenden Prozentwerte: $51 \%$ Ski und 34\% Snowboard. Wohl sind die Snowboardfahrer und -fahrerinnen eine Minderheit, doch bei den Jugendlichen verliert der Ski gegenüber dem Snowboard an Popularität. Eine der großen Schwierigkeiten besteht darin, daß somit Informationen nicht nur über Klimaänderung und das künftige Klima benötigt werden, sondern auch solche über den zukünftigen Tourismus. Es müssen somit Szenarien oder Zukunftsbilder des Tourismus entworfen werden. Wer weiß aber schon, wie im Jahre 2030/2050/2100 der Tourismus aussehen wird?

Damit ist auch ein Hauptproblem der Klimafolgenforschung angesprochen, nämlich die Unsicherheit, die Ungewißheit. HENDERSON-SELLERS (1993) verwendet den treffenden Ausdruck einer Kaskade der Unsicherheit («cascade of uncertainty»). Das heisst, die Unsicherheit nimmt von allgemeinen Zirkulationsmodellen (GCMs) über regionale und lokale Klimamodelle bis hin zu Impaktmodellen ständig zu. Diese Kaskade der Unsicherheit bildet zweifellos eine der Hauptschwierigkeiten, wenn es darum geht, die Ergebnisse von Klimafolgenforschungen in der (touristischen) Praxis umzusetzen.

Ferner werden beim interaktiven Ansatz auch Anpassungsstrategien der touristischen Anbieter und Nachfrager mit einbezogen. Auf seiten der Anbieter wird beispielsweise die künstliche Beschneiung forciert, Skigebiete werden, sofern es die topographische Situation erlaubt, nach oben erweitert, oder Skilifte werden durch bodenunabhängige Transportanlagen (Sessellifte) ersetzt. Eine Umfrage bei den Verkehrsvereinen im Kanton Graubünden zeigte, daß eine klare Mehrheit den vermehrten Einsatz von Beschneiungsanlagen und die Umstellung auf bodenunabhängige Transportanlagen befürwortet. Pistenplanierungen - planierte Pisten benötigen eine geringere Mächtigkeit der Schneedecke - und die Hochgebirgserschließung sind (vorläufig noch?) eher verpönt.

Bei einer Erhöhung der Durchschnittstemperatur um 2 Grad Celsius dürfen in der Schweiz nur noch Skigebiete über $1500 \mathrm{~m}$ ü.M. als schneesicher bezeichnet werden, wobei auch hier zwischen 1500 und $2000 \mathrm{~m}$ ü. M. mit einem reduzierten Pistenangebot zu rechnen ist. Von den 230 Skigebieten in der Schweiz dürfen unter heutigen Bedingungen 195 oder $85 \%$ als schneesicher bezeichnet werden. Bei einer Temperaturerhöhung um 2 Grad Celsius werden es noch 144 oder $63 \%$ sein; 94 davon befinden sich in den Kantonen Wallis und Graubünden. Für einzelne Skigebiete kann somit eine Erhöhung der Durchschnittstemperatur, sofern die nicht temperaturabhängigen Voraussetzungen gegeben sind, zur Folge haben, daß die Anzahl Skifahrer zunehmen wird. Dies wiederum kann zu Kapazitätsproblemen führen, was in einer weiteren Phase, sofern das Skigebiet nicht weiter ausgebaut wird oder aus Gründen des Landschaftsschutzes werden kann, eine Verringerung der Anzahl Skifahrer bewirkt.

Noch wesentlich anspruchsvoller ist der integrative Ansatz («integrated approach»). Bei diesem werden die Auswirkungen einer Klimaänderung nicht nur auf einen einzelnen Sektor, auf ein Teilsystem, beispielsweise den Tourismus, betrachtet, sondern auf ein gesamtes sozioökonomisches System, woraus dann wiederum Aussagen für einen bestimmten Sektor abgeleitet werden können. Welche Auswirkungen besitzen eine Klimaänderung und andere Bestimmungsgrößen auf die Berglandwirtschaft, auf die Forstwirtschaft usw., und wie wirken sich diese wiederum auf den Tourismus und umgekehrt - aus? Integrativ bedeutet ferner, daß die komplexen Beziehungen zwischen der lokalen, regionalen, nationalen und globalen Betrachtungsebene mit berücksichtigt werden müssen. Dies kann an zwei einfachen Beispielen dargelegt werden:

1. Die Anzahl Tage mit Schneebedeckung im schweizerischen Mittelland hat in den letzten Jahren und Jahrzehnten deutlich abgenommen. Die Tourismusverantwortlichen in den Skigebieten befürchten nun, daß die potentiellen Nachfrager die Beziehung zu Kälte und Schnee verlieren und deshalb im Winter weniger Skiferien buchen. Das heißt, die Nachfrage nach Skifahren wird nicht nur durch das Vorhandensein von Schnee in den Zielgebieten, sondern auch in den Quellgebieten mitbestimmt.

2. Immer mehr Personen verbringen ihre Winterferien nicht in den Bergen, sondern in Feriendestinationen am Meer (Karibik, Indik, Rotes Meer, Kanarische Inseln) dies nicht zuletzt auch als Folge tiefer Flugpreise. Eine repräsentative Umfrage für die Wintersaison 1997/98 (LINK-Umfrage) zeigt, daß 29\% der befragten Schweizerinnen und Schweizer Wintersportferien in den Bergen verbringen, $5 \%$ fahren in den Süden, $57 \%$ verbringen keine Wintersportferien, und die restlichen $9 \%$ wissen nicht, ob, und wenn ja, wo sie ihre Wintersportferien verbringen werden. Wie wirkt sich nun eine Klimaänderung (z. B. Erhöhung des Meeresspiegels) auf diese Feriendestinationen im Süden aus, und was hat dies allenfalls für Rückwirkungen auf den Wintertourismus in den Bergen? Das heißt, es gilt nicht nur die Folgen der Klimaänderung im eigentlichen Untersuchungsgebiet, sondern auch in Konkurrenzgebieten abzuschätzen, inklusive der komplexen Wechselwirkungen zwischen den verschiedenen Gebieten.

Der einfache «impact approach» muß zweifellos als zu simplifizierend beurteilt werden. Dies bedeutet nicht, daß Aussagen über die Auswirkungen einer Klimaänderung auf die physikalischen Rahmenbedingungen des Tourismus, wie Dauer der Schneebedeckung, 
Schneehöhe usw., vernachlässigt werden dürfen. Vielmehr müssen diese laufend überprüft, verbessert und den aktuellen Erkenntnissen der Klimaforschung angepaßt werden. Anderseits muß der integrative Ansatz als zu komplex beurteilt werden. Schon ohne Berücksichtigung der Auswirkungen einer Klimaänderung sind die Schwierigkeiten bei der Modellierung sozioökonomischer Teil- und Gesamtsysteme enorm. Realistischerweise sollten sich deshalb die Forschungsanstrengungen auf den interaktiven Ansatz konzentrieren, bei dem nicht allein die klimatischen Faktoren, sondern auch andere Bestimmungsgrößen und die dazugehörigen Rückkoppelungen sowie Anpassungsprozesse mit berücksichtigt werden.

\section{Von den wissenschaftlichen Erkenntnissen zur Umsetzung}

Ziel der Klimafolgenforschung ist, nicht nur Auswirkungen einer Klimaänderung zu analysieren, sondern auch Strategien, Maßnahmen, Empfehlungen zu entwickeln, um negative Folgen zu verringern, positive zu unterstützen. Dabei ergeben sich weitere methodische Probleme. Ein Hauptproblem stellt zweifellos die bereits erwähnte Unsicherheit, Ungewißheit dar. Die Auseinandersetzung mit den Folgen einer Klimaänderung weist sehr oft auf Probleme hin, mit denen ein Wirtschaftszweig auch ohne Klimaänderung, teilweise allein wegen der heutigen natürlichen Variabilität des Klimas, konfrontiert ist. Im alpinen Tourismus ist es beispielsweise die große Abhängigkeit vom Wintertourismus und damit die wintertouristische Monostruktur zahlreicher inneralpiner Regionen. Es gilt deshalb, Strategien und Maßnahmen, sog. «no regrets», zu entwickeln, die zur Verbesserung der Situation beitragen, auch wenn die Klimaänderung nicht oder nicht im vorgesehenen Ausmaß stattfinden sollte. Solche Strategien sind auch leichter umzusetzen, da die Zahl der Entscheidungsträger, die eine Klimaänderung als nicht sehr relevant betrachten oder sie gar bezweifeln, nicht unterschätzt werden darf. Strategien lassen sich ferner unterteilen in Anpassungsstrategien und Vermeidungsstrategien. Erstere, touristische Beispiele wurden weiter oben genannt, haben zum Ziel, die (wirtschaftliche) Bedeutung des untersuchten Sektors auch unter veränderten Klimabedingungen zu erhalten und allenfalls gar zu vergrößern. Vermeidungsstrategien dienen dazu, das Ausmaß und die Geschwindigkeit der Klimaänderung zu reduzieren. Dies kann nur geschehen, wenn die Emissionen klimarelevanter Gase, vor allem von Kohlendioxid, verringert werden. Im Tourismus geht es um eine Optimierung des Energieeinsatzes durch die konsequente Ausnutzung der Energiesparpotentiale und durch den vermehrten Einsatz von Alternativenergie sowie um Maßnahmen im Bereich Verkehr. Vermeidung bedeutet aber auch, da $\beta$ eine Branche alle Maßnahmen, die sie betreffen, nicht allein bezüglich ihrer Umwelt- und Sozialverträglichkeit, sondern auch hinsichtlich der Klimaverträglichkeit überprüfen muß. Das heisst, es geht in Ergänzung eher kurzfristiger Strategien um den Einsatz für eine langfristige Umwelt- und Klimapolitik.

Entscheidend für den Erfolg von Anpassungs- und Vermeidungsstrategien ist, daß diese nicht von der Wissenschaft allein, sondern in enger Zusammenarbeit mit den Entscheidungsträgern und der Öffentlichkeit erarbeitet werden. Dabei spielen Kenntnisse über die Wahrnehmung der Klimaänderung eine Schlüsselrolle. In einer Arbeit über mögliche Auswirkungen des Klimawandels aus Sicht der Bergsteiger wurde untersucht, wie die bereits heute erkennbaren naturräumlichen Veränderungen, beispielsweise der Gletscherschwund, wahrgenommen werden, worauf diese zurückgeführt werden und welche Auswirkungen sie auf das Bergsteigen haben. 95 Prozent der befragten Bergführer und 70 Prozent der übrigen Berggänger sind der Meinung, daß eine Klimaänderung Auswirkungen auf das Bergsteigen haben wird. Man befürchtet einen weiteren Rückzug der Gletscher und eine Zunahme der Steinschlaggefahr. Die zu erwartenden Entwicklungen lassen vermuten, daß die Klimaänderung mit einer Zunahme der Gefahren und der alpintechnischen Schwierigkeiten einhergeht. Die Alpinisten müssen sich also mit einem Szenario «Gefahrenzunahme» auseinandersetzen. In der Untersuchung wurden die befragten Personen mit diesem Szenario konfrontiert und auf allfällige Reaktionen getestet. Drei Viertel der Bergführer wollen im Fall einer Gefahrenzunahme weitermachen wie bisher, kaum einer beabsichtigt, seinen Beruf aufzugeben. Bei den übrigen Berggängern sieht es ähnlich aus. Auch diese Gruppe ist im Fall einer Gefahrenzunahme nicht bereit, auf die Ausübung ihrer Freizeitaktivität zu verzichten. Der Anteil derjenigen, die weitermachen würden wie bisher, ist allerdings kleiner als bei den Bergführern. Dies ist nicht weiter verwunderlich, handelt es sich doch beim Bergsteigen im einen Fall um den Broterwerb, im anderen aber nur um eine Freizeitbeschäftigung.

In diesem Zusammenhang ist auf das Arbeiten mit und in sog. «focus groups» hinzuweisen. Es handelt sich dabei um Gruppendiskussionen mit Entscheidungsträgern, im vorliegenden Fall mit Tourismusverantwortlichen, und Betroffenen. Durch die Arbeit in Gruppen verspricht man sich bezüglich der Sensibilisierung von Touristikern und Touristen für die Probleme und Folgen des Klimawandels einen besseren Erfolg als in Einzelinterviews und -gesprächen. Das Ziel solcher Gruppendiskussionen ist, zu besseren Kenntnissen über die Wahrnehmung der Klimaänderung zu gelangen, ferner Strategien und Maßnahmen aus Sicht der Tourismusverantwortlichen und Betroffenen kennenzulernen. Damit können Strategien und Maßnahmen, die im Rahmen der Klimaänderungsfolgenforschung erarbeitet werden, besser bezüglich ihres Stellenwertes, ihrer Realitätsnähe und ihrer Umsetzbarkeit beurteilt werden. Es geht schließlich um das gemeinsame Erarbeiten von $\mathrm{Zu}$ kunftsbildern des Tourismus - vor dem Hintergrund veränderter Klimabedingungen. 


\section{Fazit}

Die Folgen von Klimaänderungen hängen nicht nuur von klimatischen Entwicklungen ab, sondern auch davon, wie auf Klimaänderungen von Betroffenen und Entscheidungsträgern reagiert wird. Bei der Klimafolgenforschung bzw. Klimaänderungsfolgenforschung handelt es sich um ein junges Forschungsgebiet, das sich in den nächsten Jahren in theoretischer, methodischer und empirischer Hinsicht stark weiterentwickeln wird. Eine engagierte, gesellschaftsrelevante Geographie muß ihr fachliches Potential hier einbringen, denn, wie dies der Klimaschutzbeauftragte der Stadt Hamburg prägnant formuliert hat, «die Menschen, die in potentiell betroffenen Regionen leben oder in potentiell betroffenen Wirtschaftszweigen arbeiten, wollen wissen, was globale Klimaänderungen für sie bedeuten können. Planer und Entscheidungsträger wollen wissen, womit sie rechnen müssen, und diejenigen, die über politische Maßnahmen zum Schutz des Klimas entscheiden, wollen wissen, welche Folgen ihre Entscheidungen und auch welche Folgen unterlassene Entscheidungen haben können» (KRUPP, 1995, S. 13).

\section{Literatur}

ABEGG, B. (1996): Klimaänderung und Tourismus: Klimafolgenforschung am Beispiel des Wintertourismus in den Schweizer Alpen. Schlußbericht NFP 31, vdf Zürich.
ABEGG, B., KÖNIG, U., BÜRKI, R., ELSASSER, H. (1997): Climate Impact Assessment im Tourismus. Die Erde, 128: 105-116. Englische Übersetzung in: Institute for Scientific Cooperation (ed.) (1998): Applied Geography and Development. A Biannual Collection of Recent German Contributions Vol. 51: 81-93. Tübingen.

HENDERSON-SELLERS, A. (1993): An Antipodean Climate of Uncertainty? Climatic Change, 25: 203-224.

LOHMANN, M., et al. (1998): Küstentourismus in Deutschland: Nachfragestruktur und die Anfälligkeit für Klimaänderungen. Tourismus Journal H. 1, Bd. 2: 67-79.

MORGAN, D. L. (1997): Focus Groups as Qualitative Research. Qualitative Research Methods Series Vol. 16. 2nd ed., Thousand Oaks.

KÖNIG, U. (1998): Tourism in a Warmer World: Implications of Climate Change Due to Enhanced Greenhouse Effect for the Ski Industry in the Australian Alps. Wirtschaftsgeographie und Raumplanung, Vol. 28, Zürich.

KRUEGER, R. A. (1994): Focus Groups - A Practical Guide for Applied Research. 2nd ed., Thousand Oaks.

KRUPP, Ch. (1995): Klimaänderungen und die Folgen - Eine exemplarische Fallstudie über die Möglichkeiten und Grenzen einer interdisziplinären Klimafolgenforschung, Berlin.

Programmleitung NFP 31 (Hrsg.) (1996): Klimarisiken - Herausforderung für die Schweizer Wirtschaft. Thesen zu Klimaänderungen und Naturkatastrophen. Arbeitsbericht NFP 31, vdf Zürich.

SCHWÖRER, D.-A. (1997): Bergführer und Klimaänderung. Eine Untersuchung im Berninagebiet über mögliche Auswirkungen einer Klimaänderung auf den Bergführerberuf. Sargans.

STERR, H. (1993): Klimawandel, Klimawirkungsforschung und die Rolle der Geographie. Geographische Rundschau 3/45: 196-198.

TIMMERMAN, P. (1989): Everything else will not remain equal: The Challenge of Social Research in the Face of a Global Climate Warming. In: Impacts of Climate Change on the Great Lake Bassin (61-76). Report of the first US - Canada Symposium on Impacts of Climate Change on the Great Lake Bassin, 27-29 September 1988, Oak Brook, Illinois. 